\section{Roll up for the revolution}

\author{
In the early 1990s, a \\ chance finding in a \\ Japanese laboratory \\ introduced the world to \\ carbon nanotubes. \\ Today, interest in the \\ tubes is still growing. \\ Philip Ball reports on a \\ decade of discovery.
}

$\mathrm{T}$ years ago this month, Sumio Iijima, a microscopist at the NEC Corporation laboratories in Tsukuba, Japan, described how he found a needle in a haystack that was to revolutionize nanoscale science. Iijima's hollow needle, which had formed on the end of a graphite electrode, was just a few nanometres wide, several micrometres long, and made of pure carbon. It was a carbon nanotube ${ }^{1}$

A decade on, nanotubes have accumulated an impressive list of real and potential applications. They have improved the precision of scanning probe microscopes ${ }^{2}$, and formed the basis for nanoscale tweezers that can pick up and move tiny particles ${ }^{3}$. In the past few months alone, logic gates ${ }^{4,5}$, the building blocks of computers, have been made from nanotubes, raising hopes that they could be used to create electronic circuits or even whole computers. "Nanotubes will be cheap, environmentally friendly, and do wonders for humankind," says Richard Smalley of Rice University in Houston, Texas.

Carbon nanotubes arrived at a time when physicists and chemists were exploring a new form of carbon - the cage-like fullerene molecules discovered in 1985 by Harry Kroto of the University of Sussex in England in collaboration with Smalley's team in Houston ${ }^{6}$. In graphite, carbon exists as flat sheets, with all the atoms arranged in a hexagonal pattern. But in fullerenes, there are also pentagons, so a sheet of atoms can form a hollow sphere.
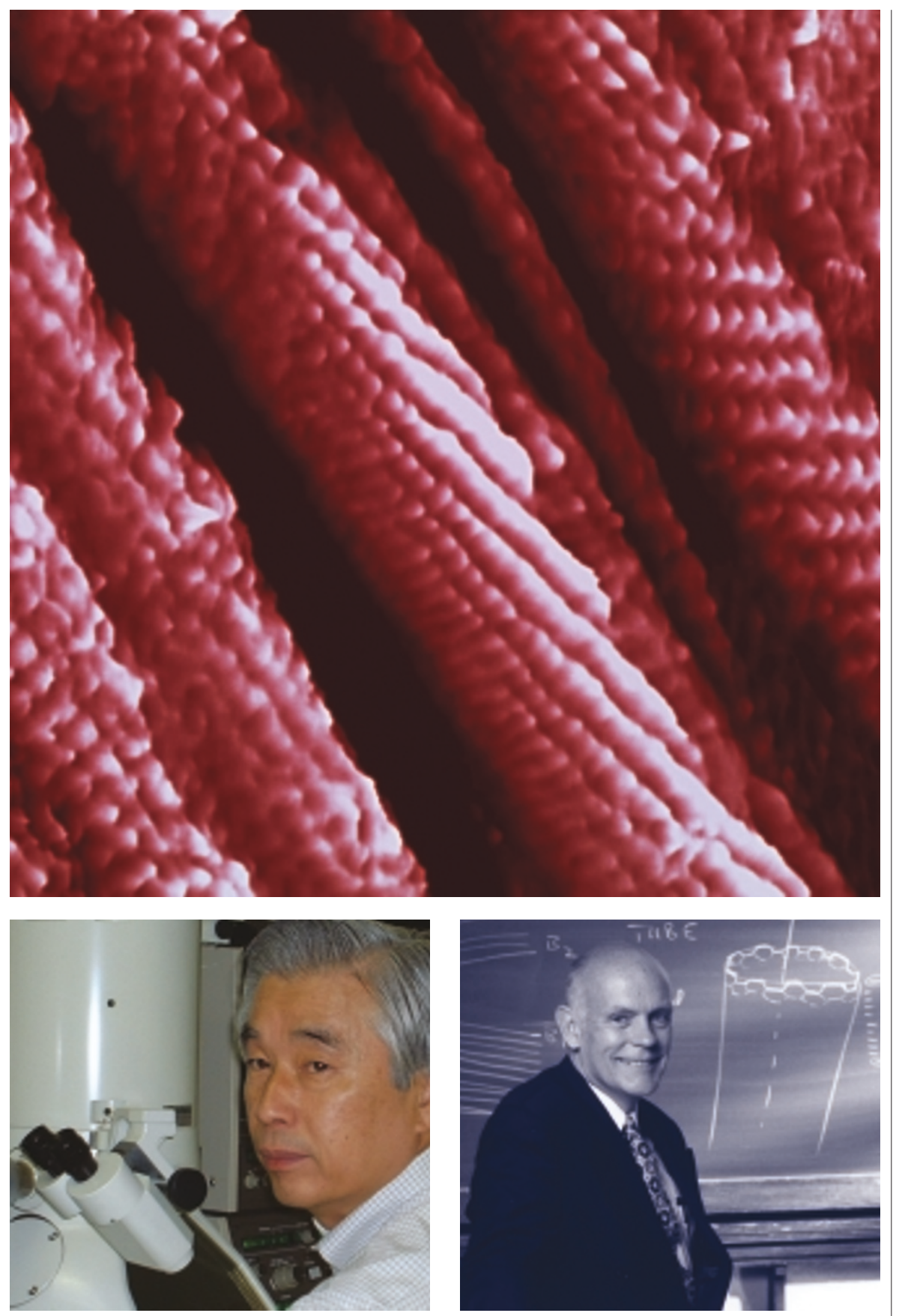

In the pipeline: Richard Smalley (right) believes that carbon nanotubes (top), which were brought to the world's attention by Sumio Iijima (left), will be cheap and environmentally friendly materials.

In 1990, a team led by Wolfgang Krätschmer at the Max Planck Institute for Nuclear Physics in Heidelberg, Germany, and Donald Huffman of the University of Arizona in Tucson showed that fullerenes could be mass-produced by passing an electric current between two touching graphite electrodes surrounded by helium ${ }^{7}$. The heat produced vaporizes the graphite, and fullerenes form as the gaseous carbon cools.

Iijima had been studying carbon-fibre production, which uses similar methods, since the 1980s. Inspired by the mass production study, he tinkered with Krätschmer and Huffman's experimental conditions. Rather than bringing the two electrodes together,
Iijima kept them a short distance apart, so that sparks passed between them. To his surprise, he found fine carbon needles - nanotubes - growing on the negative electrode.

The nanotubes looked like sheets of graphite rolled up into tubes, with the ends closed off by caps of various shapes. But unlike fullerenes, the tubes were made up of a series of cylinders nestling one inside the other like Russian dolls — referred to as multiwalled tubes. There is now evidence that Iijima was not the first to discover carbon nanotubes (see 'Who saw the first nanotube?', overleaf), but after his 1991 paper, researchers woke up to the tubes' potential for the first time.

Initially, the multiwalled tubes posed a 


\section{news feature}

problem for theorists, who struggled to predict their physical and electrical properties. But the simpler structure of single-walled tubes was easier for calculations, and the predictions provided much of the impetus for subsequent research. According to the theorists, single-walled tubes would be extremely stiff and would behave as either metallic conductors or as semiconductors, depending on how the rows of hexagons were orientated relative to the tube's length. Multiwalled tubes were thought to contain a mixture of both types, but their single-walled counterparts offered the chance to study the 'pure' electrical behaviour.

But it wasn't until 1993 that researchers were able to make such tubes. Iijima's team ${ }^{8}$ and Donald Bethune's group at IBM's Almaden Research Center in San Jose, California ${ }^{9}$, simultaneously discovered that the growth of single-walled nanotubes is promoted by mixing cobalt or iron into the graphite electrodes.

\section{Carbon connections}

Electrical conductivity was perhaps the tubes' most enticing property. Commercial techniques for making silicon chips can currently create wires about 180 nanometres wide. If nanotubes could be used as wires, this width would be reduced by at least an order of magnitude. In addition, the promise that some tubes would behave as semiconductors led researchers to suspect that they could be used to form nanoscale versions of components such as transistors - electronic devices that can switch or amplify current. This year, nanotubes have even been shown to behave as superconductors, materials that lose all of their electrical resistance below a certain transition temperature. So far, the highest transition temperature for nanotubes is $15 \mathrm{~K}$ (ref. 10), which is too low for practical applications.

Hongjie Dai and his colleagues at Stanford University took a step towards electronic circuits based on nanotubes when, in 1998 , they showed how individual single-walled nanotubes might be grown directly onto a simple electrical circuit ${ }^{11}$. Dai's nanotubes formed between metallic islands on a silicon surface as heat was used to break down the methane gas surrounding the surface.

With modification, nanotubes can also become electrical devices in their own right. In 1998, Cees Dekker's group at the Delft University of Technology in the Netherlands turned a nanotube into a transistor ${ }^{12}$. Dekker passed current through the nanotube using metal electrodes attached to each end of the tube. A voltage applied to a third electrode underneath the tube altered the way in which the nanotube conducted electricity, and could be used to switch current flowing through the nanotube on and off. Other groups have since produced different types of nanotube transistor.
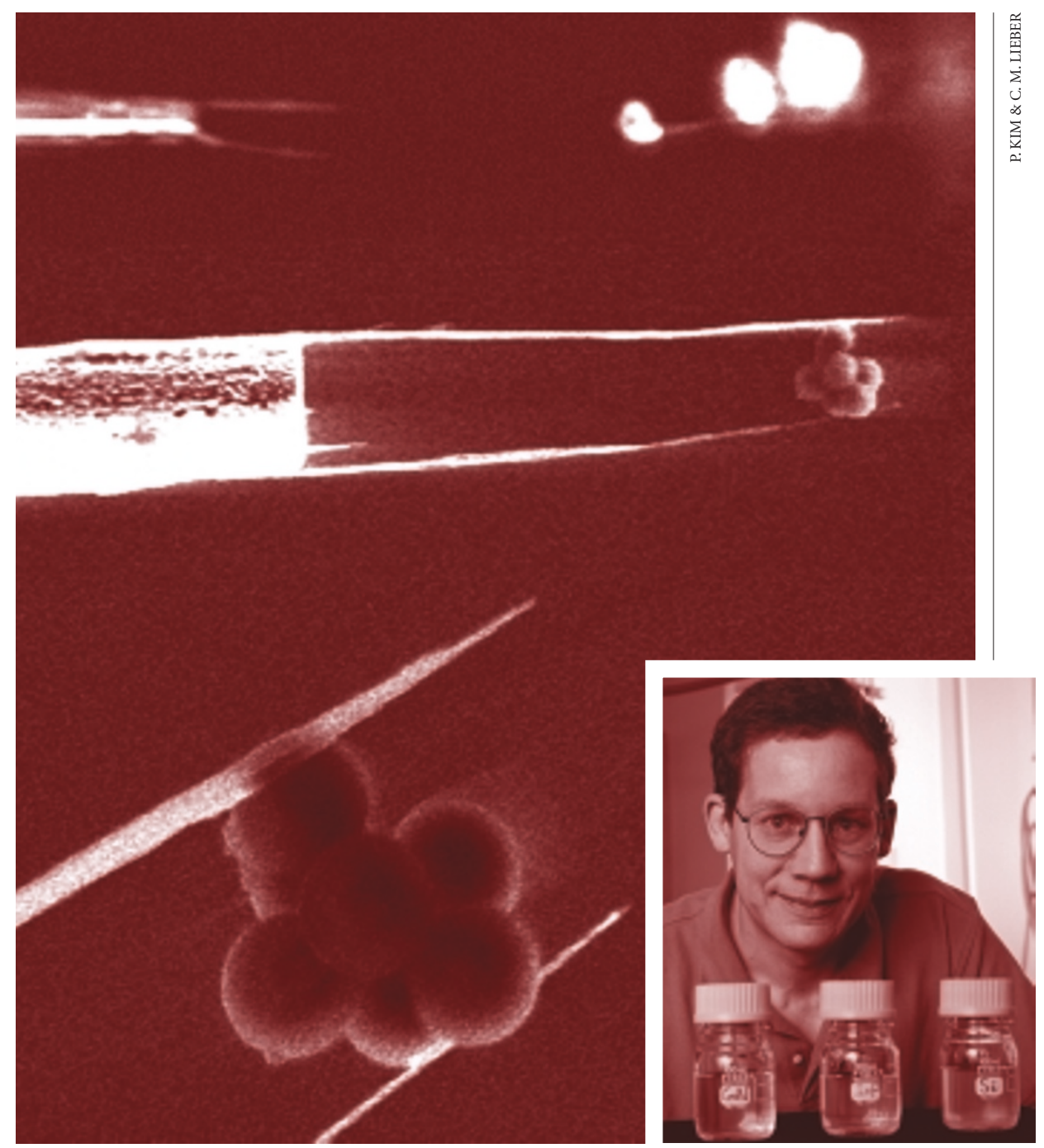

In a pinch: Charles Lieber (inset) has made nanotube 'tweezers', shown here picking up 350-nm beads.

Transistors are the basic components in logic gates, the electronic devices used to build computers. This August, Phaedon Avouris and his colleagues at IBM's Thomas J. Watson Research Center in Yorktown, New York, described how, by wiring two types of nanotube transistors together, they created the simplest type of logic gate - one that inverts its input, turning a binary ' 1 ' into a ' 0 ' and a ' 0 ' into a ' 1 ' (ref. 4). Dekker's group, meanwhile, has made gates with other, more complex behaviour ${ }^{5}$.

\section{Uphill struggle}

Despite the promise of this work, conventional silicon technologies are so well established that replacing them will be difficult "I would not expect to see the commercial use of nanotubes in logic or computer applications within this decade," Dekker says. In addition, cheap production of nanotube-based circuits might depend on other technologies, such as molecular selfassembly $^{13}$, which have yet to mature.

Researchers are also getting excited about the mechanical properties of nanotubes Single-walled tubes are very strong, and are stiffer than the conventional carbon fibres currently used in composite materials. They can also be bent double and yet spring back into shape without suffering any apparent damage.

Such properties have led to some surprising applications. For example, the resolution of scanning probe microscopes — which provide images of a surface by moving a fine tip across it - depends on the sharpness of the probe's tip. And the longer the tip, the more deeply the microscope can probe into surface cavities. In 1996, Smalley and colleagues showed that mounting a nanotube on the microscope's tip improves both resolution and penetration ${ }^{2}$ - and the tube's strength means that it will simply bend, instead of snapping, if it is pushed a little too far into the surface. Charles Lieber's group at Harvard University has since extended the concept by adding chemical groups to the end of the tips, so that the microscope can recognize the chemical composition of the surface being studied ${ }^{14}$. Further work indicates that a wide range of different substances, including biological molecules, can be attached to the tubes ${ }^{15,16}$. 
Together with his colleague Philip Kim, who is now at Columbia University in New York, Lieber has also used nanotubes as extremely fine tweezers ${ }^{3}$. They attached two nanotubes to a glass rod patterned with two electrodes. Giving these electrodes different charges creates an electrostatic attraction between the nanotubes, which makes them bend towards each other. This allowed Lieber and Kim to use the tubes to pick up and move tiny polymer beads. A team led by Yoshikazu Nakayama at Osaka Prefecture University in Japan has recently created similar tweezers on the tip of a scanning probe microscope ${ }^{17}$, raising the possibility that the microscope could be used both to observe and to manipulate samples, such as individual cells.

Exciting as these projects are, all exploit the physical properties of nanotubes at the microscopic scale. Materials scientists would like to take advantage of these properties over longer length scales, but at present, nanotubes cannot be grown longer than a millimetre or so. Last year, a team led by Philippe Poulin of the University of Bordeaux in France created fibres and ribbons from a tangled web of nanotubes ${ }^{18}$; but the tubes were not continuous, so the ribbons lacked the strength of individual nanotubes.

Nevertheless, commercial applications are emerging. In 1999, Jong-min Kim and his colleagues at the Samsung Advanced Institute of Technology in Suwon, Korea, developed a flatscreen colour display in which nanotubes supply the electron beams that cause phosphors on the screen to light up ${ }^{19}$.

\section{Screen shots}

The nanotubes act as a crucial component of miniature cathode-ray tubes - the bulky devices used in conventional televisions. Strong electric fields are used to pull electrons from the tips of the nanotubes, and the electrons are focused into a beam by tiny electrodes. Similar devices have been under development for many years, using electron emitters made from semiconductors or diamond. But nanotubes have sharper ends than the tips that can be made from these other materials - and the sharper the emitter's tip, the lower the electric field needed to extract electrons.

For such applications to be commercially viable, nanotubes will have to be readily available and inexpensive. Last year Smalley co-founded Carbon Nanotechnologies Incorporated (CNI), a Houston-based company geared to producing nanotubes. This September, CNI set to work on a new manufacturing system to produce 200 grams

\section{Who saw the first nanotube?}

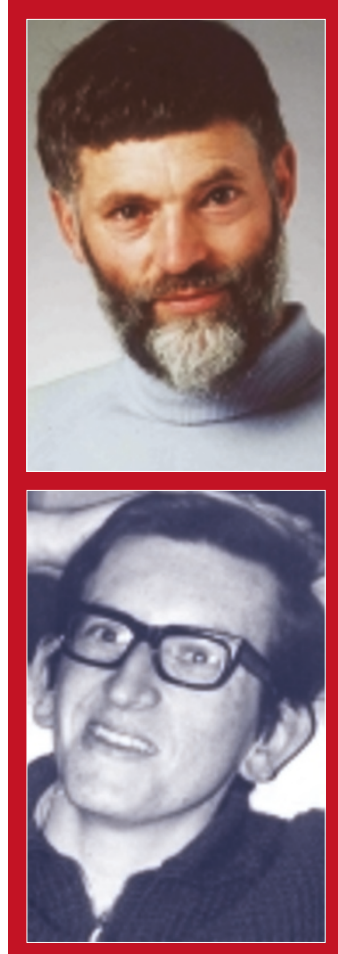

The history of carbon nanotubes may be a little longer than was previously thought. Richard Smalley of Rice University in Houston, Texas, points out that nanotubes could have been unknowingly produced by late nineteenth century chemists experimenting on methane ${ }^{21}$. And in the 1960 s and 1970 s, at least two groups made and characterized the tubes, only for their discoveries to go largely unnoticed.

Roger Bacon of the National Carbon Company in Parma, Ohio, then part of Union Carbide, produced nanoscale scrolls of graphite in 1960 (ref. 22), confirming their dimensions and structure using microscopy and $X$-ray diffraction. Bacon's work may have been ignored by science historians, says Smalley, because of the view that he made scrolls. But recent work ${ }^{23}$ has shown that Sumio lijima at NEC in Tsukuba, Japan, who in 1991 published what is often thought to be the first paper on nanotubes, was using a procedure that generates a mixture of scrolls and tubes, suggesting that Bacon may also have done this.

In the late 1970s, Peter Wiles (bottom, left) and John Abrahamson (top, left) of the University of Canterbury in Christchurch, New Zealand, went one step further. They were investigating the carbon fibres produced when sparks were passed between two graphite electrodes, and found that one of the electrodes was coated with "mats of small fibres"24. In 1979, electrondiffraction measurements showed that the walls of these 'fibres' were made of carbon in a graphite-like arrangement. They described the tubes as made of several layers of crystalline carbon "wrapped together" — they had discovered multiwalled carbon nanotubes.

Wiles and Abrahamson presented their findings at the 14th Biennial Conference on Carbon, held at Pennsylvania State University in June 1979. An extended abstract of that presentation was published at the time ${ }^{25}$, but no further reports appeared in print. The contrast with the excited reception of lijima's paper is remarkable, and illustrates how new discoveries rely on the prevailing research climate. In an age before nanotechnology and the fullerene-induced interest in carbon chemistry, there seemed little reason to regard the fibres as anything more than a smaller versions of familiar micrometre-scale carbon fibres. "Although we figured we had found something novel, we never for a moment imagined the eventual significance of the discovery," says Wiles.

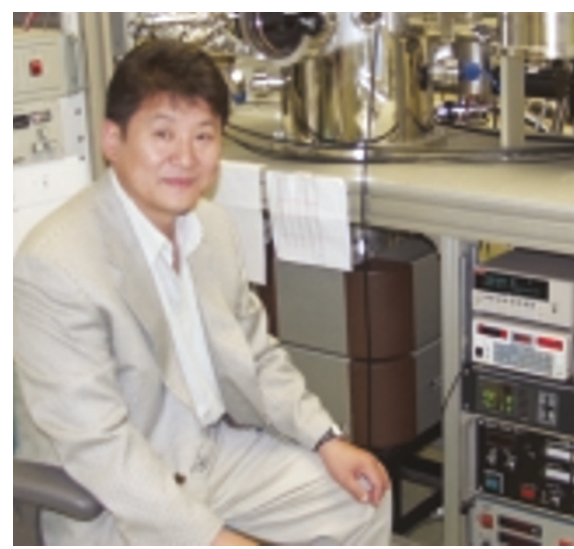

Jong-min Kim believes nanotubes could replace cathode-ray tubes to make flat TV screens.

of nanotubes per day. At around US\$500 per gram, the tubes are still not cheap, but mass production should drive costs down. CNI hopes to make around 9 kilograms a day by 2002, and could be turning out thousands of kilograms per week by 2004. "In time, millions of tonnes of nanotubes will be produced worldwide every year," predicts Smalley.

As applications and commercial uses for nanotubes begin to emerge, demand is sure to rise. And research in the field continues unabated - futuristic applications, including fuel tanks for future hydrogen-powered vehicles ${ }^{20}$ are under investigation. Some may well fall by the wayside, but it now seems inconceivable that Iijima's discovery will not make its impact felt beyond the laboratory. Philip Ball is a consultant editor for Nature.

1. Iijima, S. Nature 354, 56-58 (1991).

2. Dai, H., Hafner, J. H., Rinzler, A. G., Colbert, D. T. \& Smalley, R. E. Nature 384, 147-150 (1996)

3. Kim, P. \& Lieber, C. M. Science 286, 2148-2150 (1999).

4. Derycke, V., Martel, R., Appenzeller, J. \& Avouris, Ph. Nano Lett. 1, 453-456 (2001)

5. Bachtold, A., Hadley, P., Nakanishi, T. \& Dekker, C. Science 4 October 2001 (10.1126/science.1065824).

6. Kroto, H. W., Heath, J. R., O'Brien, S. C., Curl, R. F. \& Smalley, R. E. Nature 318, 162-163 (1985).

Krätschmer, W., Lamb, L. D, Fostiropoulos K. \& Huffman, D. R. Nature 347, 354-358 (1990).

8. Iijima, S. \& Ichihashi, T. Nature 363, 603-605 (1993).

9. Bethune, D. S. et al. Nature 363, 605-607 (1993).

10. Tang, Z. K. et al. Science 292, 2462-2465 (2001).

11. Kong, J., Soh, H. T., Cassell, A. M., Quate, C. F. \& Dai, H., Nature 395, 878-881 (1998)

12. Tans, S. J., Verschueren, A. R. M. \& Dekker, C. Nature 393, 49-52 (1998).

13. Ball, P. Nature 413, 667-668 (2001).

14. Wong, S. S., Joselevich, E., Woolley, A. T., Cheung, C. L. \& Lieber, C. M. Nature 394, 52-55 (1998).

15. Erlanger, B. F., Chen, B.-X., Zhu, M. \& Brus, L. Nano Lett. 1, 465-467 (2001)

16. Star, A. et al. Angew. Chem. Int. Ed. 40, 1721-1725 (2001).

17. Akita, S. et al. Appl. Phys Lett. 79, 1691-1693 (2001).

18. Vigolo, B. et al. Science 290, 1331-1334 (2000).

19. Choi, W. B. et al. Appl. Phys Lett. 75, 3129-3131 (1999)

20. Zandonella, C. Nature 410, 734-735 (2001).

21. Hughes, T. V. \& Chambers, C. R. US Patent 405,480 (1889).

22. Bacon, R. J. Appl. Phys. 31, 283 (1960).

23. Mordkovich, V. Z. et al. in Supercarbon: Synthesis, Properties,

and Applications (eds Yoshimura, S. \& Chang, R. P. H.) 107-118 (Springer, Berlin, 1998).

24. Wiles, P. G. \& Abrahamson, J. Carbon 16, 341-349 (1978).

25. Abrahamson, J., Wiles, P. G. \& Rhoades, B. L. Abstract in

proceedings of the 14th Biennial Conf. On Carbon,

Pennsylvania State Univ., June 1979. Reprinted in Carbon 37 1873-1874 (1999). 